\title{
Imagem Cardiovascular em Pacientes com COVID-19
}

\section{Cardiovascular Imaging in COVID-19}

\author{
Rujittika Mungmungpuntitpantip ${ }^{1}$ e Viroj Wiwanitkit ${ }^{1}$ \\ Dr. DY Patil University, ${ }^{1}$ Pune - Índia
}

\section{Caro Editor,}

Gostaríamos de compartilhar ideias relacionadas à publicação "Imagem Cardiovascular em Pacientes com COVID-19."1 Grossman e Lima concluíram que "cardiologistas nucleares e médicos nucleares devem estar cientes dos achados incidentais em pacientes assintomáticos com COVID-19 e devem otimizar os protocolos de CPM, quando o procedimento for necessário."1 Os resultados desse estudo estão de acordo com um relato anterior da Ásia. ${ }^{2}$ A imagem pode ajudar a identificar problemas cardíacos e pulmonares que podem ocorrer de modo assintomático devido à COVID-19 ou a uma patologia silenciosa anterior. ${ }^{2} \mathrm{Um}$ ponto importante é o diagnóstico diferencial da lesão nova e da patologia anterior subjacente. Nos países tropicais, pode existir uma patologia comum, como a tuberculose, que resulta em dificuldades de interpretação de novos problemas cardíacos e pulmonares devido à COVID-19. ${ }^{3}$ Considerando-se que a interpretação das imagens depende principalmente do radiologista, torna-se necessário que os radiologistas tenham maior consciência e cuidado ao interpretar as imagens clínicas durante a pandemia de COVID-19.

\section{Palavras-chave}

Doenças Cardiovasculares; Pneumopatias; Coronavírus; COVID-19; Pandemia; Diagnóstico por Imagem; Pacientes Assimtomáticos.

Correspondência: Rujittika Mungmungpuntitpantip •

Private Academic Consultant, Bangkok Thailand bangkok 1039 - Tailândia

E-mail: rujittika@gmail.com

Artigo recebido em 22/12/2020, revisado em 10/02/2021,

aceito em 10/02/2021

DOI: https://doi.org/10.36660/abc.20201356

\section{Referências}

1. Grossman GB, Lima RL. Cardiovascular Imaging in Patients with COVID-19. Arq Bras Cardiol. 2020 Nov; 115(5):973-4.

2. Attavirayanuparuktham B. Abnormal heart imaging in COVID-19 patients: a note. Adv Lab Med Int. 2020;10:18-9.
3. Yasri S, Wiwanitkit V. Tuberculosis and novel Wuhan coronavirus infection: Pathological interrelationship. Indian J Tuberc. 2020 Apr;67(2):264. 